\title{
Uterine Atony: An Innovative Dutta's Scoring System for Elective Cesarean Section
}

\author{
${ }^{1}$ Dilip Kumar Dutta, ${ }^{2}$ Indranil Dutta
}

\begin{abstract}
Uterine atony appears suddenly and is mostly unpredictable and accounts for $80 \%$ of causes of postpartum hemorrhage $(\mathrm{PPH})$, it is also one of the important causes of maternal death.

Objective: To analyze the efficacy of Dutta's score for early diagnosis and management of uterine atony during elective lower segment cesarean section (LSCS) to prevent PPH.
\end{abstract}

Study methods: This study was undertaken at JNM, NSGH, CN at Kalyani, Nadia, West Bengal, India, from 1st June 2008 to 31st Dec 2012. Six hundred cases undergoing elective LSCS were selected for randomized trial. Clinical observations were made after placental expulsion for scoring which includes shape and size of uterus, rugosity, tone, placental localization and time of placental expulsion. Scores of 0, 1, 2 were given on each observation. Three groups are created depending on scoring: group $A(n=300)-8$ to 10 , group $B(n=220)-5$ to 7 and group $C(n=80)-<5$ for better management.

Management protocols were formulated in the three groups for prevention of PPH: group A-oxytocin $10 \mathrm{U}$ (5U IM + 5U IV $30 \mathrm{drops} / \mathrm{min}$ in Ringer's lactate $500 \mathrm{ml}$ ), group B-oxytocin $15 \mathrm{U}$ (5U IM + $10 \mathrm{U}$ IV 30 drops/min in Ringer's-lactate $500 \mathrm{ml}$ ) + methylergometrine $(0.25 \mathrm{mg} \mathrm{IM})+$ anterior posterior uterine wall compression, group $\mathrm{C}$-oxytocin $20 \mathrm{U}(5 \mathrm{U}$ IM plus $15 \mathrm{U}$ IV 30 drops in ringer lactate $500 \mathrm{ml})+$ methylergometrine $(0.5$ $\mathrm{mg}$ IM) + carboprost $(250 \mathrm{mcg}$ IM) + lateral followed by anterior posterior uterine wall compression + isthmus compression of uterus + misoprostol $800 \mathrm{mg}$ per rectal during postoperative period.

Results: After adopting Dutta's score and management protocols, it was interesting to observe that intra and postoperative, blood loss (within 2 hours) were found to be significantly reduced in group A-73.3\% (<300 cc), 88\% (<200 cc) and group B-63.6\% (<300 cc), 81.8\% (<200 cc). Intraoperative blood loss $>500 \mathrm{cc}$ were also found to be reduce in group A-14.7\%, group B-16.3\%. In group C, intraoperative blood loss $>500$ cc were seen in $41.25 \%$ and postoperative $-37.5 \%$ respectively. Hemoglobin level below $11 \mathrm{gm} \%$ were found to be not reduced in group A (1.4\%), group B $(2.6 \%)$ and group $C(8.7 \%)$ after 24 hours of delivery hemodynamic status

\footnotetext{
${ }^{1}$ Senior Consultant, ${ }^{2}$ Assistant Professor

${ }^{1}$ Department of Obstetrics and Gynecology, Gice Hospital Nadia, West Bengal, India

${ }^{2}$ Department of Obstetrics and Gynecology, IQ City Medical College, Durgapur, West Bengal, India

Corresponding Author: Dilip Kumar Dutta, Senior Consultant Department of Obstetrics and Gynecology, Gice Hospital Nadia, West Bengal, India, Phone: +919051556424, e-mail: drindranildutta@yahoo.com
}

in all groups were found to be uneventful. Maternal mortality was found to be nil.

Conclusion: Early diagnosis and management of uterine atony during elective LSCS after adopting Dutta's score were found to be not only reduce intra- and postoperative blood loss but also was found to maintain a satisfactory hemoglobin level and hemodynamic status. Maternal mortality was found to be nil. This randomized trial highlighted the importance of prompt treatment in group $C$ to reduce intra- and postoperative blood loss and maternal mobidity and mortality.

Keywords: Dutta's score, MMR, PPH, Pregnancy scoring, Uterine atony.

How to cite this article: Dutta DK, Dutta I. Uterine Atony: An Innovative Dutta's Scoring System for Elective Cesarean Section. J South Asian Feder Obst Gynae 2015;7(3):113-117.

Source of support: Nil

Conflict of interest: None

Date of received: 11 May 2015

Date of acceptance: 29 July 2015

Date of publication: December 2015

\section{INTRODUCTION}

Paramount importance for every obstetrician is to prevent postpartum hemorrhage (PPH) due to uterine atony during elective lower segment cesarean section (LSCS) by early detection, assessment of the severity and search for specific causes. There may be a clinical scenario which mainly requires consideration is: (1) PPH after delivery of baby, (2) PPH after delivery of placenta and (3) secondary PPH.

Considering above facts, randomized trial was undertaken to review and analyze early diagnosis and management of uterine atony during elective LSCS in an uncomplicated pregnancy to prevent $\mathrm{PPH}$ by advocating Dutta's score.

\section{MATERIALS AND METHODS}

This randomized trial was undertaken at JNM, NSGH, $\mathrm{CN}$ at Kalyani, Nadia, West Bengal, India, from 1st June 2008 to 31st Dec 2012. Six hundred cases, who undergone elective LSCS, were selected for this study and elective cases were selected randomly. Primigravida, 18 to 30 years, 38 to 40 weeks maturity were selected for this randomized trial. 
During LSCS, clinical observations were advocated on findings of uterine condition after placental expulsion by control cord traction for scoring which includes shape and size, rugosity, tone, placental localization and time of placental expulsion. Score of 0, 1, 2 were given on each observation. Three groups are created: group A (300) - 8 to 10 , group $B(n=220)-5$ to 7 and group $C(n=80)-<5$ for prompt management. Management protocols were formulated in the three groups for preventing PPH as follows:

1. Group A: Oxytocin $10 \mathrm{U}$ (5U IM + 5 U IV $30 \mathrm{drops} / \mathrm{min}$ in Ringer Lactate $500 \mathrm{ml}$ ).

2. Group B: Oxytocin $15 \mathrm{U}$ ( $5 \mathrm{U}$ IM $+10 \mathrm{U}$ IV 30 drops/min in Ringer's lactate $500 \mathrm{ml})+$ methylergometrine $(0.25$ mg IM) + anterior-posterior uterine wall compression.

3. Group C: Oxytocin $20 \mathrm{U}$ (5U IM plus $15 \mathrm{U}$ IV 30 drops in ringer lactate $500 \mathrm{ml})+$ methylergometrine $(0.5 \mathrm{mg} \mathrm{IM})+$ carboprost (250 mcg IM) + lateral followed by anterior-posterior uterine wall compression + isthmus compression of uterus + misoprostol 800 mg per rectal during postoperative period.

Postoperatively, oxytocin $5 \mathrm{U}, 30$ drops in DNS for 4 hours in group A, 5 unit for 6 hours in DNS in group B, $10 \mathrm{U}$ in divided doses for 12 hours RL and DNS in group $\mathrm{C}$ were administered depending on findings on vaginal bleeding and uterine contraction. Continuous bladder drainage by indwelling catheter for 24 hours were advocated in all groups.

- Thorough history taking, clinical assessment, blood profile were done.

- Ultrasonography was advocated to see fetal parameter and placental localization, its shape and size, etc.

- Primigravida, 18 to 30 years, 38 to 40 weeks maturity were selected for this randomized trial.

- One thousand milliliters crystalloid was given before regional spinal anesthesia (bupivacaine $2.5 \mathrm{cc}$ ) to prevent hypovolemia.
- Blood sugar in between 100 and $120 \mathrm{mg}$, normal level of $\mathrm{Na}, \mathrm{Ca}, \mathrm{K}, \mathrm{Mg}$ and platelet count above 2 lakh were maintained 12 hours before LSCS.

- After placental expulsion uterus pulled out exteriorly for quick diagnosis. Closure of uterine wound were done in two layers with absorbable chromic 1 suture after securing bleeding in the uterine cavity and ensuring absence of blood clots inside the uterine cavity. Total time taken is 10 to 15 minutes. Uterus is then replaced in the original anatomical position in the abdomen.

- Intraoperative blood loss was estimated from standard mop $(50 \times 20 ")$ weight, blood from suction apparatus and blood clot.

- Postoperative blood loss per vagina was estimated by collecting blood in kidney tray and weight of sanitary pad.

- Informed consent was obtained from the patient and relatives.

\section{Exclusion Criteria}

Congenital anomaly of uterus, patient in labor, prolonged and obstructed labor, malnutrition, any medical and surgical complications of pregnancy, twin or triplet pregnancy, hydramnios, fibroid uterus, etc.

\section{OBSERVATIONS}

Showing criteria of scoring depending on observations on uterus following placental expulsion-shape and size of uterus, rugosity, tone, placental localization and time of placental expulsion (Table 1).

Distribution of groups, pattern of scoring and score were advocated for better management (Table 2).

Different type of protocols along with drugs, doses, routes, etc. were advocated in three groups for better management (Table 3).

Table 1: Criteria of scoring

\begin{tabular}{llll}
\hline Uterus & 0 & 1 & 2 \\
\hline Shape and size & Broad and flat discoid & $\begin{array}{l}\text { Less elevated, narrow, hard and } \\
\text { globular shape }\end{array}$ & $\begin{array}{l}\text { More elevated, narrow } \\
\text { hard and globular shape }\end{array}$ \\
Rugosity & Absent & Present in anterior or posterior surface & Present in both surfaces \\
Tone & Soft & Firm & Hard, contracted \\
Placental localization & Lower segment & Fundo anterior & Fundo posterior \\
Time of placental expulsion & $>5$ minutes & $3-5$ minutes & $<3$ minutes \\
\hline
\end{tabular}

Table 2: Distribution of scoring in groups

\begin{tabular}{|c|c|c|}
\hline Groups & Pattern of scoring & Score \\
\hline$A(n=300)$ & $\begin{array}{l}\text { Shape and size }(S S)-2 \text {, rugosity }(R)-2 \text {, tone }(T)-2 \text {, } \\
\text { placental localization }(P L)-1 \text { to } 2 \text {, placental expulsion }(P E)-1 \text { to } 2\end{array}$ & 8 to 10 \\
\hline$B(n=220)$ & Shape and size $(S S)-1$, rugosity $(R)-1$, tone $(T)-1$, placental localization $(P L)-1$ to 2 , placental expulsion (PE) -1 to 2 & 5 to 7 \\
\hline$C(n=80)$ & Shape and size $(S S)-0$, rugosity $(R)-1$, tone $(T)-0$, placental localization $(P L)-1$ to 2 , placental expulsion (PE) -1 to 2 & $<5$ \\
\hline
\end{tabular}


Table 3: Management protocols

\begin{tabular}{|c|c|c|c|c|c|c|c|}
\hline Groups & Oxyt $t^{*}$ & Methylerg ${ }^{* *}$ & Carboprost & $\begin{array}{l}\text { Lateral compression } \\
\text { on upper segment of } \\
\text { uterine wall }\end{array}$ & $\begin{array}{l}\text { Anterior-posterior } \\
\text { uterine wall } \\
\text { compression }\end{array}$ & $\begin{array}{l}\text { Isthmus } \\
\text { compression }\end{array}$ & $\begin{array}{l}\text { Misoprostol } 800 \mathrm{mcg} \\
\text { per rectal }\end{array}$ \\
\hline$A$ & $10 U$ & - & - & - & - & - & - \\
\hline B & $15 U$ & $0.25 \mathrm{mg}$ & - & - & Yes & - & - \\
\hline C & $20 U$ & $0.5 \mathrm{mg}$ & $250 \mathrm{mcg}$ & Yes & Yes & Yes & Yes \\
\hline
\end{tabular}

*Oxyt: Oxytocin; ** Methylerg: Methylergometrine

Intraoperative blood loss $<300 \mathrm{cc}$ was found to be significantly reduced in group $\mathrm{A}-73.3 \%$ and group B-63.6\%. Intraoperative blood loss $>500$ cc were reduced in group $\mathrm{A}-4.7 \%$, group $\mathrm{B}-16.3 \%$. In group C-intraoperative blood loss $>500 \mathrm{cc}$ were seen in $41.25 \%$ cases (Table 4).

Postoperative blood loss within 2 hours was found to be less $<200$ cc in $88 \%$ (group A), $81.8 \%$ in group B as compared to $25 \%$ in group C. In group C, blood loss in between 201 and $500 \mathrm{cc}$ was found to be more- $37.5 \%$ compared to group $\mathrm{A}-12 \%$ and group $\mathrm{B}-18.2 \%$. Blood loss above $500 \mathrm{cc}$ was seen in group $\mathrm{C}$ managed by blood transfusion (Table 5).

Hemoglobin level, after 24 hours, below $11 \mathrm{gm} \%$ were found to be less reduced in group A (1.4\%), group B (2.6\%) and group $\mathrm{C}(8.7 \%)$ from preoperative to postoperative period (Table 6).

Hemodynamic status in all groups was found to be uneventful except hypotension observed mainly in group $\mathrm{C}$ which was managed by replacement by fluid. No maternal death was reported (Table 7).

\section{DISCUSSION}

It is very much paramount importance for every obstetrician is to observe condition of uterus and to assess the severity and specific causes that lead to uterine atony during elective LSCS.

Evidence-based study showed that exogenous oxytocin is a strong uterotonic agent, induces the rapid onset of strong rhythmic uterine contractions mainly on upper uterine segment which last for 15 to 20 minutes and its action on gravid uterus in late pregnancy may be due to fall in the level of oxytocinase, ${ }^{1,2}$ rise of estrogen ${ }^{3}$ and fall of progesterone level. ${ }^{4}$ It was also important to know that excessive use of oxytocin produces a transient vascular smooth muscle relaxant effect may lead to mild brief reduction in blood pressure because of the reduced total peripheral resistance.

A randomized trial was undertaken by author for early detection of uterine atony and its immediate management during elective LSCS to prevent intra- and postpartum hemorrhage. It is very much important to maintain normal electrolyte balance $(\mathrm{Na}, \mathrm{Ca}, \mathrm{K}, \mathrm{Mg})$, blood sugar (100-120 mg) and platelet count $>2$ lac before advocating LSCS. ${ }^{5,6}$

Oxytocin, used in all groups in increasing dose (IM and IV) depending on scoring, is the recommended drug for prevention of $\mathrm{PPH}$ in cesarean section. ${ }^{7}$ A systematic review $^{8,9}$ of 39 trials including more than 7,900 women addresses different drugs, route, doses and time for

Table 4: Intraoperative blood loss

\begin{tabular}{llll}
\hline Groups & $<300 c c$ & $301-500 c c$ & $>500 c c$ \\
\hline A $(n=300)$ & $220(73.3 \%)$ & $66(22 \%)$ & $14(4.7 \%)$ \\
B $(n=220)$ & $140(63.6 \%)$ & $44(20 \%)$ & $36(16.3 \%)$ \\
C $(n=80)$ & $11(13.7 \%)$ & $36(45 \%)$ & $33(41.25 \%)$ \\
\hline
\end{tabular}

Table 5: Postoperative blood loss within 2 hours

\begin{tabular}{llll}
\hline Groups & $<200 c c$ & $201-500 c c$ & $>500 c c$ \\
\hline A $(\mathrm{n}=300)$ & $264(88 \%)$ & $36(12 \%)$ & - \\
B $(\mathrm{n}=220)$ & $180(81.8 \%)$ & $40(18.2 \%)$ & - \\
C $(\mathrm{n}=80)$ & $20(25 \%)$ & $30(37.5 \%)$ & $30(37.5 \%)$ \\
\hline
\end{tabular}

Table 6: Hemoglobin level in gm\%

\begin{tabular}{|c|c|c|c|c|}
\hline \multirow[b]{2}{*}{ Groups } & \multicolumn{2}{|c|}{ Preoperative } & \multicolumn{2}{|c|}{ Postoperative } \\
\hline & $<11 \mathrm{gm} \%$ & $>11 \mathrm{gm} \%$ & $<11 \mathrm{gm} \%$ & $>11 \mathrm{gm} \%$ \\
\hline$A(n=300)$ & $232(77.3 \%)$ & $68(22.7 \%)$ & $236(78.7 \%)$ & $64(21.3 \%)$ \\
\hline$B(n=220)$ & $144(65.5 \%)$ & $76(34.5 \%)$ & $150(68.1 \%)$ & $70(33.9 \%)$ \\
\hline$C(n=80)$ & $58(72.5 \%)$ & $22(27.5 \%)$ & $65(81.3 \%)$ & $15(18.7 \%)$ \\
\hline
\end{tabular}

Table 7: Hemodynamic status and maternal death

\begin{tabular}{llllll}
\hline Groups & Hypotension & Cardiac arrest & Pulmonary edema & Renal failure & Maternal death \\
\hline A & $2(0.6 \%)$ & - & - & - & - \\
B & $3(1.3 \%)$ & - & - & - & - \\
C & $5(6.3 \%)$ & - & - & - & - \\
\hline
\end{tabular}


Flow Chart 1: Management protocols to prevent $\mathrm{PPH}$ due to uterine atony during elective cesarean section

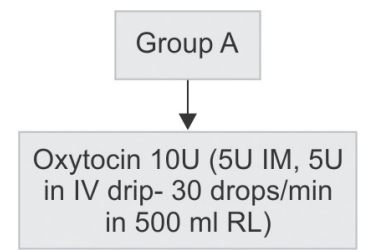

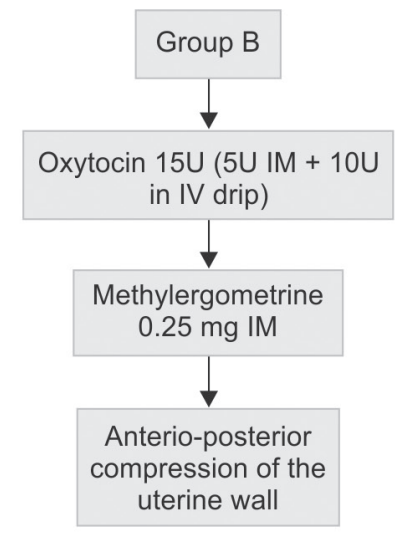

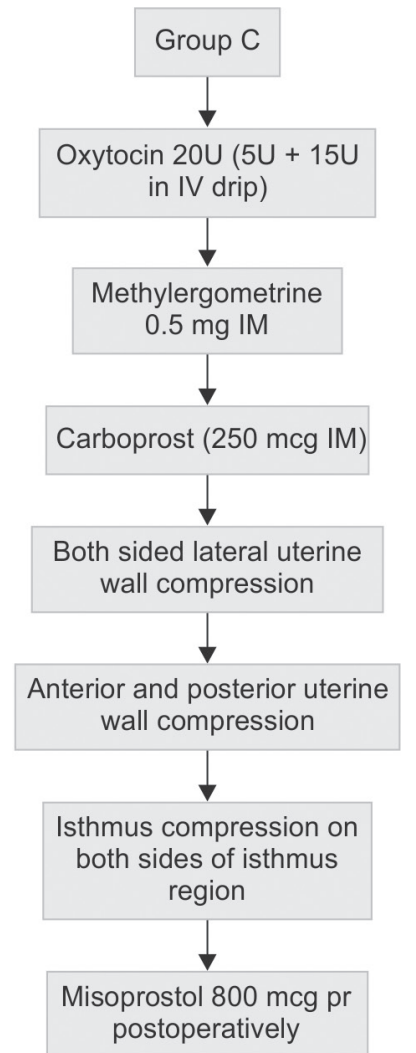

preventing PPH at both elective and emergency cesarean section, were found to be reduce the need for additional uterotonics agents which was observed in group A but does not affect the overall occurrence of major obstetrical hemorrhage as seen in groups B and C. In group B, additional use of methylergometrine $0.25 \mathrm{mg}$ IM produces prolonged uterine contractions in both upper and lower uterine segments with a duration of 60 to 120 minutes in a normotensive women; whereas in group C, methylergometrine $0.5 \mathrm{mg}$ IM and in addition injection of carboprost $250 \mathrm{mcg}$ IM were found to be a strong uterotonic effect with a duration of action is up to 6 hours. ${ }^{10}$

Anterio-posterior uterine wall compression for 2 to 5 minutes were found to be effective in group $B$, whereas in group $C$ at first lateral compression for 1 to 2 minutes to be followed by anterior-posterior compression 2 to 3 minutes, and isthmus compression (compression of both uterine arteries at the isthmus region) were found to be effective. Misoprostol $800 \mathrm{mcg}$ per rectal during postoperative period were found to be additional advantages to reduce intra- and postoperative blood loss as it was observed in this study.

It is very much significant to observe that after adopting Dutta's score and subsequent management-intraand postoperative blood loss within 2 hours were found to significantly reduce in group A-73.3\% (<300 cc), 88\% $(<200 \mathrm{cc})$ and group B-63.6\% (<300 cc), 81.8\% (<200 cc). Intraoperative blood loss $>500 \mathrm{cc}$ were also found to be reduce in group $\mathrm{A}-14.7 \%$ group $\mathrm{B}-16.3 \%$. In group $\mathrm{C}$ blood loss > 500 cc during operation- $41.2 \%$ and postoperative blood loss-37.5\% were found to be increased, as compared to groups A and B.

This study also showed that hemoglobin level below $11 \mathrm{gm} \%$ were not found to be reduced significantly either in group A (1.4\%), group B (2.6\%) and group C (5\%). Hemodynamic effects ${ }^{11,12}$ in the form of hypotension were observed in group A (6\%), group B (1.3\%) and group C (6.25\%) which were controlled by immediate management. Pulmonary edema, cardiac arrest, renal failure and maternal death ${ }^{13}$ were found to be absent. A Flow Chart 1 has been made to show the series of process to be followed as explained below.

\section{CONCLUSION}

Early diagnosis and management of uterine atony during elective LSCS, after advocating Dutta's score, were found to be not only reduce intra- and postoperative blood loss but also showed to maintain good hemoglobin level and hemodynamic status. Maternal death was found to be absent.

This randomized trial has also highlighted the importance of prompt treatment in group $C$ to reduce intra- and postoperative blood loss and maternal death.

It also appears from this trial that Dutta's scoring system is helpful in early diagnosis and treatment of primary PPH during elective LSCS. Further study may 
be multicentric will give more insight regarding the scoring system.

Flow Chart 1 created depending on the scoring system $0,1,2$ after observation of uterus following placental expulsion which includes: (1) shape and size, (2) rugosity, (3) tone, (4) placental localization and (5) time of placental expulsion.

Three groups are formed according to the scoring system as follows:

- Group A: 300 cases-score 8-10

- Group B: 220 cases-score 5-7

- Group C: 80 cases-below 5

\section{REFERENCES}

1. Nixon WCW, Smyth CN. J Obstet Gynaec Brit Emp 1957; 64:35.

2. Harris GW. Brit Med J 1948. p. 339.

3. Van SSG, Olivew S, Pincus G. Amer J Physiol 1938;4:121:98.

4. Jeffcoate TNA. Lancet 1940;1:1945.

5. Hawkins DF, Nixon WCW. J Obstet Gynaec Brit Emp 1957;64:641.
6. Corner GW, Csapo A. Brit Med J 1953;I:687.

7. Anorlu RI, Maholwana B, Hofmeyr GJ. Methods of delivering the placenta at cesarean section. Cochrane Database Syst Rev 2008 July.

8. Mahomed K, Sheehan S, Murphy DJ, Heatley E, Middleton P. Medical methods for preventing blood loss at cesarean section. Cochrane Datadase of systematic Review 2011;Dic Editorial process. Art No: CD007576. DOI:10.1002/14651858. CD007576.

9. Sarna MC, Soni AK, Gomez M, Oriol NE. Intravenous oxytocin in patients undergoing elective cesarean section. Anesth Analg 1997;84:753-756 (PubMed).

10. Svanstrom MC, Biber B, Hanes M, Johansson G, Naslund U, Balfors EM. Signs of myometrial ischemia after injection of oxytocin: a randomized double-blind comparison of oxytocin and methylergometrine during caesarean section. Br J Anaesth 2008 May;100(5):683-689.

11. Thomas JS, Koh SH, Cooper GM. Haemodynamic effects of oxytocin on infusion on women undergoing cesarean section. Br J Anaesth 2007;98:116-119.

12. Peterson M. Cardiovascular effects of oxytocin. Prog Brain Res 2002;139:281-288.

13. Cooper GM, Lewis G, Neilson J. Confidential enquiries into maternal deaths, 1997-1999. Br J Anaesth 2002;89:369-372. 